\title{
Construction of a NI ELVIS II+ Based Comprehensive Communication Circuits Experiment Platform
}

\author{
Jianfang Ye \\ Dept. of Communication Engineering \\ Donghua University \\ Shanghai, China \\ leaf6411@dhu.edu.cn
}

\author{
Jianwei Ye \\ PLA Computer Technical Service Centre \\ Guangzhou, China \\ Yejianwei1@126.com
}

\author{
Jiale Tu \\ PLA Computer Technical Service Centre \\ Guangzhou, China \\ tujiale@tom.com
}

\begin{abstract}
Practicality plays key roles in communication circuits course education. This paper addressed the development of a NI ELVIS II+ based experiment platform. This platform incorporated a large-area breadboard, a function generator, a portable power supply and an easy-to-use software interface. Students only need to get access to standard power outlets and PCs that are capable of running LabView (Student Edition) to carry out circuits design, construction, simulation, and test. The key feature is that we can bring the hands-on experiment into classroom through the Internet. When compared with traditional lecture-only courses, this unit makes the theoretical learning incorporated with the hands-on experiments. The students found the education style to be especially effective, which would stimulate their desire to learn. The result of this work applied clear merits to the idea that the comprehensive laboratory platform would greatly enhance learning experience in electrical and computer engineering curriculum.
\end{abstract}

Keywords-Communication circuits; Laboratory platform; Practice teaching; NI ELVIS

\section{INTRODUCTION}

"Communication Circuits" is the key course for students majoring in electronic and information engineering. It not only relies on the basic courses including "Analogue Electrical Circuits”, "Digital Electrical Circuits” and etc., but also lays foundation for subsequent course "Principle of Communication" and even higher leveled course "Radio Frequency and Microwave Circuits". It is an important, useful and complex course, but it is hard for teachers to teach and hard for students to learn. The course has strong practicability in engineering, and the education method for relevant experiment has a strong impact on education effect. Up till now, almost all the colleges and universities allocate relevant experiment for the course, but there is a general phenomenon that the experiment method is relatively monotonous and it mainly focuses on verification experiments. Via verification experiments, students' intuitive understanding of basic knowledge and theories from textbook can be reinforced. They can also master the basic operation method of electronic measurement instrument including function generators, scanners and oscilloscopes. However, verification experiments cannot effectively cultivate students' capability of engineering design end actual problem solving. The development and application of NI ELVIS act as the complementary part of current education experiment system, which is mainly focused on verification experiments. By adding comprehensive experiment design and combining key knowledge points with engineering design method, students can integrate the knowledge they learn. It would also help student to develop creative thoughts and cultivate their innovation and engineering design capability. By highlighting the training of the methods, principles and means of students' scientific research, the ultimate goal of encouraging students' practice and cultivating and reinforcing students' innovation awareness can finally be achieved.

\section{COMPREHENSIVE COMMUNICATION CIRCUITS EXPERIMENTAL PLATFORM}

Most of the current experiment facilities are electrical board modules including fixed chips and components. Longtime use of these facilities would result in increasing failure rate and it costs lab staff a lot of time and money for maintenance and replacement, which leads to high maintenance cost. Besides, new technologies and chips cannot be incorporated in those fixed electrical circuit modules, which leaves a gap between the content of experimental training and industry development. In order to solve the problem mentioned above, we developed the innovative and comprehensive communication circuit experiment platform based on NI ELVIS. Fig. 1 gives system structure of the comprehensive experiment platform. 


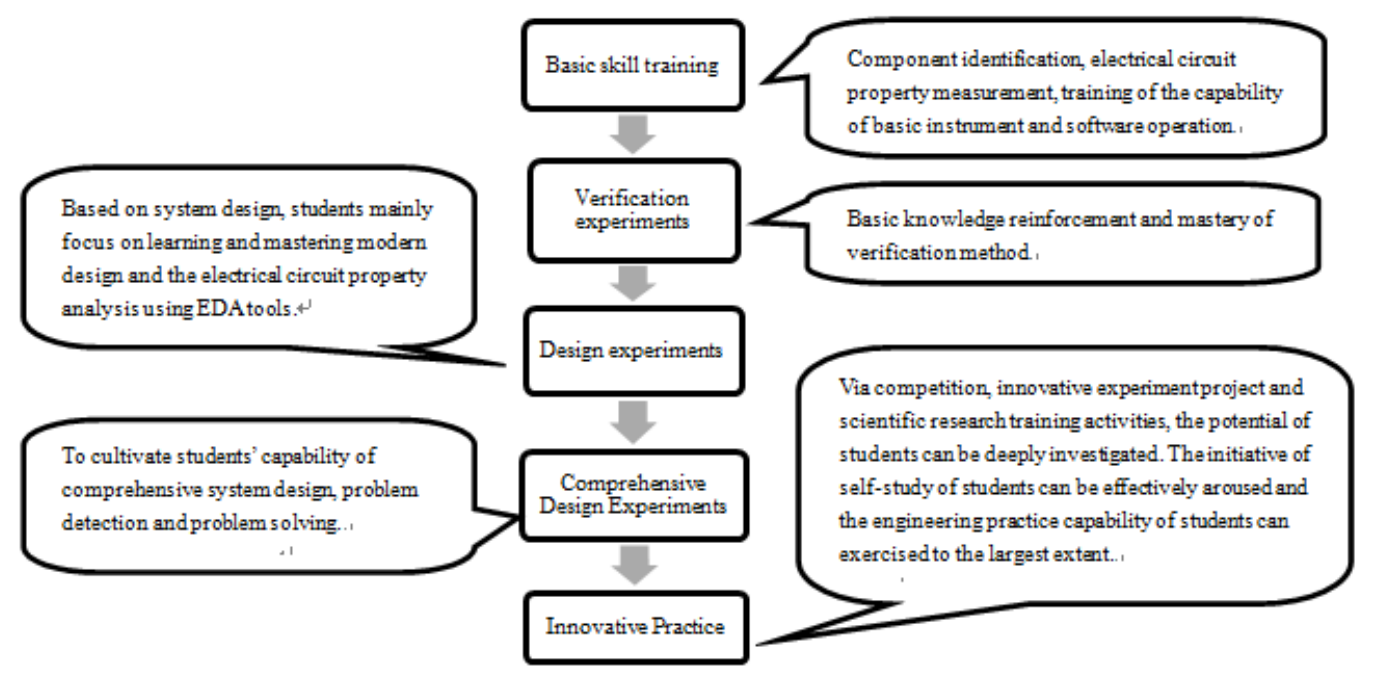

Figure 1. System structure of the comprehensive experiment platform

\section{A. System Architecture}

Fig. 2 gives basic components of the comprehensive experiment platform.

The innovative and comprehensive communication electrical circuit experiment platform based on NI ELVIS II+ includes 3 practical sectors of professional experiment education system structure, including comprehensive design and innovation aspects. It realizes the goal of highlighting profession characteristics, reinforcing students' practicability and comprehensively improves the cultivation of practical talents and innovative talents. Another key feature of the experiment platform is its openness. Teachers can incorporate the new technologies and chips in communication and electrical technology field into experiments according to different education goal and complete further development of the comprehensive experiment platform. It will make the education closely follow the newest development of the industry, fulfill students' requirements of different levels of relevant professional experiments, facilitate the construction and development of the course of electric and information engineering major.

\section{B. Hardware Composition of the Platform}

Fig. 3 gives the function flow chart of the comprehensive experiment platform based on the combination of simulation and prototype. This platform could realize the whole processing including theoretical knowledge education, simulation design, prototype development, property test and electronic product realization.

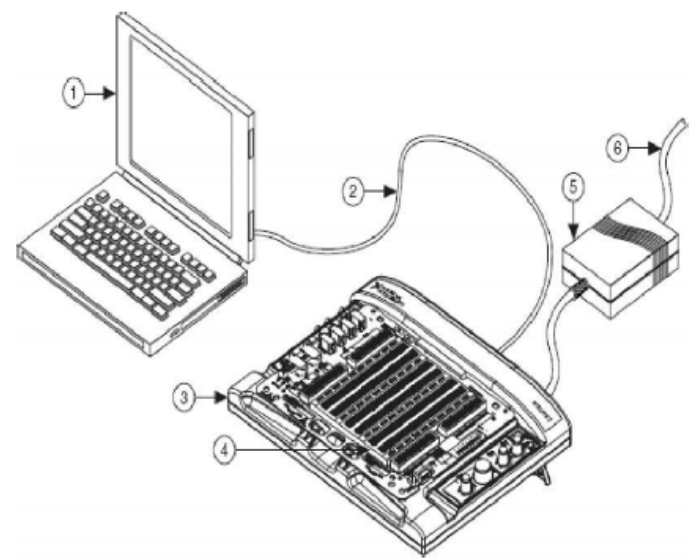

Figure 2. Comprehensive experiment platform based on the combination of simulation and prototype

\begin{tabular}{|c|c|c|c|c|}
\hline $\begin{array}{c}\text { Texlbouk } \\
\text { Theory }\end{array}$ & $\begin{array}{l}\text { Simulation and } \\
\text { Design } \\
\text { NI Multisim }\end{array}$ & $\begin{array}{l}\text { Prototype } \\
\text { Developent } \\
\text { NI ELVIS }\end{array}$ & $\begin{array}{l}\text { Test and Relization } \\
\text { LabVIEW }\end{array}$ & $\begin{array}{l}\text { PCB Products } \\
\text { Altium } \\
\text { Designer }\end{array}$ \\
\hline
\end{tabular}

Figure 3. Function flow chart of the comprehensive experiment platform 
The NI ELVIS II+ prototype unit is the key component of the comprehensive experiment platform. Hardware electrical circuit can be constructed on the breadboard of NI ELVIS II+. The built-in DAQ equipment completes the parameter collection of the electrical circuit. According to the test result of the electrical circuit property from the soft panel instrument installed in the computer, the virtual instrument based electrical circuit design, simulation analysis, hardware realization and electrical circuit test can be completed. Fig. 4 gives the development environment of the software and hardware of the platform. With the help of the platform, the interactive theoretical design, simulation analysis and the prototype hardware can be integrated and flexible shift between virtual technology and real environment can be realized. Based on the mastery of modeling method of active components, passive components and function modules in the simulation system, students can complete electrical circuit design in the simulation environment. They can adjust parameters of the electrical circuit, carry out simulation analysis upon the property of electrical circuit using Multisim, establish the dynamic connection between electrical parameters and relevant characteristics and finally use physical components to construct the actual electrical circuits on the prototype board of the NI ELVIS II+ experiment platform. With the use of the output signal waveform of the actual electrical circuit obtained from the virtual meters integrated in the platform, students can compare the simulation result with the experimental result of the actual electrical circuit using LabView Signal Express. This experiment style would allow students to spend more time on electrical circuit design and problem analysis instead of the preparation of experimental equipment and repetitive experimental data reading. It ensures the flexibility of theoretical and analytical design and greatly enhances the leaning efficiency of students. The next generation of electrical system design would involve 3G, $\mathrm{Wi}-\mathrm{Fi}$, Bluetooth, GPS wireless system, ultra-wideband technology, or even the integration of more than one technology in a single device, which is far beyond the limit that can be achieved using traditional cut and try method. With the wide application of large scale integrated circuit, the complexity of system electrical circuit keeps increasing, and it is a trend to carry out simulation analysis and optimized design with the help of EDA technology. The comprehensive experiment platform fits into this trend and incorporates the wireless communication system design and simulation analysis method.

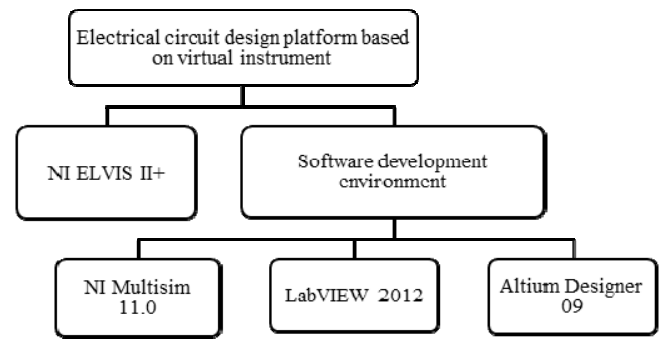

Figure 4. Development environment of of the platform
The innovative and comprehensive communication circuit experiment platform based on NI ELVIS II+ has strong extensibility. Teachers or third party manufacturers can design various kinds of experiment cards according to students' requirements and perform customized redevelopment of the ELVIS platform. The openness of the ELVIS output port effectively ensures the insertion of new experiment and the maintenance of ELVIS education platform, which is beneficial for reducing experiment cost, updating experiment content and education method according to subject development and improving education quality. The use of traditional measurement instrument in simple basic verification experiments familiarizes students with the correct operation method of basic equipment, which is the key foundation of cultivating students' engineering capabilities. In the traditional experiments, all the output experiment results are read from measurement instrument one by one by students. Improper use of equipment or error in data reading would make it impossible for students to draw correct conclusions in experimental result analysis, which severely affect the reinforcement and consolidation of basic theories and knowledge via experiment process and results as well as the realization of education goal of experiment process. Virtual instrument acts as the necessary and useful complement of traditional instrument. In complex and comprehensive design experiments, the introduction of virtual instrument provided by LabView could promptly and accurate obtain the output result of electrical circuit via the built-in DAQ equipment in ELVIS platform. It directly gives accurate waveform in the SFP of the computer interface, which relieves students from time-consuming and heavy repetitive data reading work so that they can devote precious experiment time to more innovative learning and improvement.

Besides the development of local experiment system, the remote interactive comprehensive experiment system can be constructed based on iLAB structure and Moodle technology. Fig. 5 gives the structure of the remote interactive comprehensive experiment system. The iLAB structure provides services including remote experiment access, experiment pre-registration, experiment management, user registration, login, identity verification, authorized service and daily management. The design of website hosting experiment details is based on Moodle. It provides relevant experiment preparation webpage, which allows the addition of experiment operation videos to reinforce students' understanding of experiments. In addition, the establishment of online community strengthens the communication among students and between students and teachers.

With the help of the platform, the interactive theoretical design, simulation analysis and the prototype hardware can be integrated and flexible shift between virtual technology and real environment can be realized. Based on the mastery of modeling method of active components, passive components and function modules in the simulation system, students can complete electrical circuit design in the simulation environment. They can adjust parameters of the electrical circuit, carry out simulation analysis upon the 
property of electrical circuit using Multisim, establish the dynamic connection between electrical parameters and relevant characteristics and finally use physical components to construct the actual electrical circuits on the prototype board of the NI ELVIS II+ experiment platform. With the use of the output signal waveform of the actual electrical circuit obtained from the virtual meters integrated in the platform, students can compare the simulation result with the experimental result of the actual electrical circuit using LabView Signal Express. This experiment style would allow students to spend more time on electrical circuit design and problem analysis instead of the preparation of experimental equipment and repetitive experimental data reading. It ensures the flexibility of theoretical and analytical design and greatly enhances the leaning efficiency of students. The next generation of electrical system design would involve $3 G$, Wi-Fi, Bluetooth, GPS wireless system, ultra-wideband technology, or even the integration of more than one technology in a single device, which is far beyond the limit that can be achieved using traditional cut and try method. With the wide application of large scale integrated circuit, the complexity of system electrical circuit keeps increasing, and it is a trend to carry out simulation analysis and optimized design with the help of EDA technology. The comprehensive experiment platform fits into this trend and incorporates the wireless communication system design and simulation analysis method. It develops a number of designs, comprehensive and innovative experiments, constructs a whole new experiment education system and effectively cultivates the capability of electrical circuit design and analysis of students.

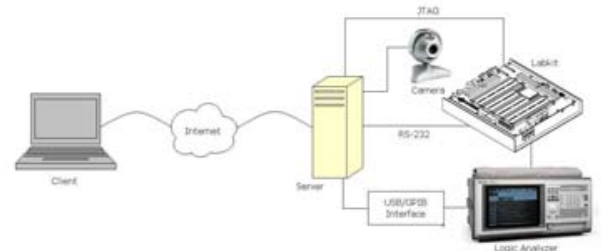

Figure 5. Structure of the remote interactive comprehensive experiment system

The most important significance of the remote interactive experiment system is the introduction of basic experiments into classroom teaching. The integration of experiment education and classroom teaching completely eliminates the gap between experiment education and classroom teaching, which restricts the education quality improving. It drastically enriches the education methods and provides substantial foundations for the development of the interactive experiment platform served for communication and electrical circuit design activities, which has a strong impact on the reinforcement of students' mastering and understanding of the key knowledge.

\section{CONCLUSION}

The realization of the platform completes two breakthroughs.

1) All the local experimental data can be entered into the computer via the built-in DAQ card in ELVIS and the analysis experiment results can be directly exported. Experiment report can be directly submitted via the network, which solves the problem to find places to store the increasing number of students' reports. Teachers only have to $\log$ in to the database of the experiment course to get to know the experiment condition of students, which realizes paperless management. Teachers can directly supervise experiments and give dynamic guidance via the network.

2) Physical instrument can be partially replaced by the virtual instrument developed by the platform, which largely reduce the hardware investment of laboratories and improve the accuracy of experimental data acquisition and experiment efficiency. A large amount of time that is devoted to redundant repetitive data reading can now be saved for deep analysis of experiment result and physical concept investigation.

With the help of the experiment platform, a number of design, comprehensive and innovation experiments can be developed to complement verification experiments, establish a whole new experimental education system and enrich experiment content and methods. It provides perfect software and hardware environment for the further development of the capability of engineering practicability and actual problem solving and cultivation of engineering technical talents. Students generally acknowledge that the effective combination of classroom studying and practice by comprehensive experiment platform improves the traditional passive learning education method and provides them with perfect hardware platform for their innovative design and greatly enhances their learning efficiency.

\section{ACKNOWLEDGMENT}

The work of this paper is part of the project "Construction of high-level engineering courses", funded by Shanghai Education Committee.

\section{REFERENCES}

[1] Gilmore R, Besser L. Modern Wireless RF Circuits Design[M. Weng, trans.]. Beijing: Electronic Industry Press; 2006

[2] Gruenbacher D, Natarajan B, Kuhn W. Work in Progress: An Integrated Laboratory Experience - A New Environment for Teaching Communications. 36th ASEE/IEEE Frontiers in Education Conference; 2006 Oct 27-31; San Diego, CA. Amsterdam: 2006. P. 78

[3] Knight D, Sullivan J, Poole S, Carlson L. Skills Assessment in Hands-On Learning and Implications for Gender Differences in Engineering Education. Annu Conf Expo. 2000 Mar;3(23):4-10

[4] Mantri A, Dutt S, Jupta P, Chitkara, M. Design and Evaluation of a PBL-based Course in Analog Electronics. IEEE Trans, Educ. 2010 Nov;51(4):432-438

[5] Mitchell J, Canavan, B, Smith, J. Problem-based learning in communication systems: student perceptions and achievement. IEEE Trans. Edu. 2010 Nov;53(4):587-594

[6] Sayre C. Complete Wireless Design. 2nd ed. New York: McGrawHill Professional; 2008 Nov 